\title{
DIFERENCIAS EN ANSIEDAD EN FUNCIÓN DEL CURSO EN UNIVERSITARIOS
}

\author{
Francisco Manuel Morales Rodríguez \\ Universidad de Granada \\ fmmorales@ugr.es \\ Ana María Morales Rodríguez \\ Universidad de Málaga \\ AMoralesR@uma.es
}

Recepción Artículo: 17mayo 2021 Admisión Evaluación: 17 mayo 2021 Informe Evaluador 1: 19 mayo 2021

Informe Evaluador 2: 23 mayo 2021

Aprobación Publicación: 01 junio 2021

\section{RESUMEN}

En esta situación de pandemia de docencia no presencial en grupos grandes, cada vez es más frecuente que el alumnado pueda sufrir estrés académico y ansiedad ante los continuos retos que ha de afrontar como los exámenes, la situación económica, la adaptación a nuevas metodologías de enseñanza/aprendizaje que requieren un mayor uso de las TIC (Tecnologías de la Información y de la Comunicación). El objetivo del presente trabajo es analizar las diferencias en ansiedad percibida en función del curso y del género en una muestra de 203 estudiantes universitarios españoles pertenecientes en su mayoría a Ciencias de la Educación y Psicología. No se encontraron diferencias estadísticamente significativas en función del género en la variable ansiedad, pero sí se encontraron diferencias estadísticamente significativas en función del curso. Se utilizó un diseño ex post facto. Los datos obtenidos son relevantes para el diseño de acciones e intervenciones psicoeducativas para la prevención del estrés y ansiedad que contribuyan a la mejora del bienestar, calidad de vida y del rendimiento académico en estudiantes universitarios.

Palabras clave: ansiedad; curso; evaluación; universitarios

\section{ABSTRACT}

Differences in anxiety as a function of course in university students. Increased non-face-to-face teaching imparted to large groups due to the ongoing pandemic has led to academic stress and anxiety becoming common among students, because of the continuous challenges they have to face, such as exams, the economic situation, and having to adapt to new teaching/learning methodologies that require greater use of ICT (Information and Communications Technology). Thus, this study, for which an ex post facto design was utilized, aimed to analyze the differences in perceived anxiety according to the course and gender among 203 Spanish university students, most of them pursuing educational sciences and psychology_statistically significant differences were 


\section{DIFERENCIAS EN ANSIEDAD EN FUNCIÓN DEL CURSO EN UNIVERSITARIOS}

observed according to the course, but not gender. The results obtained could help design relevant psychoeducational actions and interventions for the prevention of stress and anxiety, to contribute to improvement in wellbeing, quality of life, and academic performance in university students.

Keywords: anxiety; course; assessment; university students

\section{INTRODUCCIÓN}

En esta situación de pandemia de docencia no presencial en grupos grandes, cada vez es más frecuente que el alumnado pueda sufrir estrés académico y ansiedad ante las responsabilidades académicas como los exámenes y la adaptación que implica el empleo de un mayor uso de las TIC y la docencia online.

Con respecto a la definición de la ansiedad estado, Spielberger (1972) planteó que la ansiedad-estado alude a un "estado emocional" inmediato y modificable a lo largo del tiempo mientras que la ansiedad-rasgo hace referencia a tendencia o rasgo de personalidad relativamente estable.

Es importante la evaluación de este constructo que se ha asociado a los bajos niveles de autoeficacia (Domínguez-Lara, 2016; Finney y Schraw, 2003)

Castillo, Chacón y Díaz-Véliz (2016) evaluaron la ansiedad y el estrés en situaciones académicas en una muestra de 154 estudiantes pertenecientes a la Facultad de Medicina de la Universidad de Chile (de la Escuela de Enfermería y de la de Tecnología Médica), evidenciando que dichos estudiantes universitarios presentan niveles de ansiedad estado y ansiedad rasgo elevados (mayor en los que pertenecen a Enfermería) y sufren estrés académico, entre otra fuentes, por el tener que realizar un examen, por la falta de tiempo para acometer las actividades académicas y por la sobrecarga académica. En la misma línea, en otro estudio (Cardona-Arias, PérezRestrepo, Rivera-Ocampo, Gómez-Martínez y Reyes, 2015) encontraron en una muestra de 200 estudiantes universitario una prevalencia de ansiedad del $58 \%$ señalándose que están expuestos a exigencias psicológicas, académicas y sociales que hacen necesario un diagnóstico temprano para minimizar las conductas de riesgo para la salud y el fracaso académico.

Arrieta Vergara, Díaz Cárdenas y González-Martínez (2014) también encontraron una prevalencia alta de síntomas ansiosos en una muestra de 973 estudiantes universitarios con edades entre los 18 y 35 años. Concretamente en otra investigación, Riveros, Hernández y Rivera (2007) se ponen de manifiesto que entre los factores asociados a la ansiedad en el ámbito académico se encuentran las responsabilidades académicas que generan estrés y las relaciones con el resto del estudiante y docentes. Asimismo, otro estudio más reciente (Caballero-Domínguez y Suarez-Colorado, 2019) encontró en una muestra de estudiantes universitarios pertenecientes a Ciencias de la Salud diferentes tipos de respuesta ante el estrés indicándose que a mayor nivel de burnout académico mayores son los niveles de ansiedad.

Por todo lo anterior, en la actual situación con el impacto educativo y social que ha tenido la pandemia y siendo el ámbito universitario uno de los que puede generar estrés en los jóvenes, se hace necesario este tipo de estudios para evaluar los niveles de ansiedad en dicho contexto universitario con vistas al entrenamiento de aquellas estrategias de afrontamiento consideradas más productivas o eficaces.

\section{OBJETIVOS DE LA INVESTIGACIÓN}

El objetivo del presente trabajo fue analizar las diferencias en ansiedad percibida en función del curso y del género en una muestra de 203 estudiantes universitarios españoles pertenecientes en su mayoría a Ciencias de la Educación y Psicología. Se esperaba encontrar niveles de ansiedad más elevados en el primer curso que supone la entrada y adaptación al sistema universitario y el último curso donde tienen que afrontar el Trabajo Fin de Grado y las Prácticas Externas, así como otras exigencias sociales y académicas.

\section{MUESTRA Y/O PARTICIPANTES}

Los participantes fueron 203 estudiantes universitarios españoles pertenecientes en su mayoría a Ciencias de la Educación y Psicología, la mayoría mujeres. Se excluyeron los datos de las personas que no había cumpli- 
mentado todos y cada uno de los cuestionarios aplicados, eran estudiantes a tiempo parcial y personas con Necesidades Educativas Especiales. Se trabajó con una muestra incidental

\section{METODOLOGÍA Y/O INSTRUMENTOS UTILIZADOS}

\section{Diseño}

Se utilizó un diseño ex post facto. Transversal.

\section{Instrumento}

Inventario de Ansiedad Estado-Rasgo (STAI) (Fonseca-Pedrero, Paino, Sierra-Baigrie, Lemos-Giráldez y Muñiz, 2012; Spielberger, Gorsuch, Lushene y Cubero, 2011). Este instrumento está formado por 40 ítems, donde los veinte primeros evalúan la Ansiedad Estado y los otros veinte la Ansiedad Rasgo. Tiene un formato de respuesta tipo likert de tres puntos: 0 indica nada y 3 mucho. Sus propiedades psicométricas son adecuadas.

\section{Procedimiento}

El estudio contó con la aprobación del Comité de Ética en Investigación de la Universidad de Granada. Se aseguró el anonimato, la confidencialidad, la posibilidad de abandonar el estudio en cualquier momento y se recogió el consentimiento informado de los participantes en el estudio.

\section{RESULTADOS ALCANZADOS}

A continuación, se muestran en la Tabla 1 el análisis de diferencias en ansiedad estado en función del curso. En la Tabla 2 se muestra el análisis de diferencias en ansiedad rasgo en función del curso en la muestra de participantes en el estudio. No se encontraron diferencias estadísticamente significativas en función del género en la variable ansiedad, pero sí se encontraron diferencias estadísticamente significativas en función del curso. Concretamente el alumnado de cuarto curso de la presente muestra mostró mayores niveles de ansiedad rasgo y ansiedad estado.

Tabla 1. Diferencias en ansiedad en función del curso en ansiedad estado.

\begin{tabular}{lccccc}
\hline & Curso & Media & Dt & $t$ & $p$ \\
\hline Ansiedad & & & & & \\
estado & Primero & 21.8 & 9.7 & -2.85 & 0.00 \\
& Cuarto & 27.7 & 9.1 & & \\
\hline
\end{tabular}

Tabla 2. Diferencias en ansiedad en función del curso en ansiedad rasgo.

\begin{tabular}{lccccc}
\hline & Curso & Media & Dt & $t$ & $p$ \\
\hline Ansiedad & & & & & \\
rasgo & Primero & 22.8 & 9.8 & -2.41 & 0.01 \\
& Cuarto & 27.9 & 8.1 & & \\
\hline
\end{tabular}

\section{DISCUSIÓN}

Los resultados en el presente estudio exhiben que el estudiantado de cuarto curso presentó mayores niveles de ansiedad. Cabe esperar que en ese alumnado se pueda sufrir mayor ansiedad escolar considerando que en nuestro contexto el alumnado de cuarto ha tenido que afrontar sus Trabajos de Fin de Grado y sus Prácticas Externas con los cambios y adaptaciones en las adendas correspondientes para puedan ser llevadas a cabo en incluso posponer la realización de algunas de sus Prácticas por la situación de pandemia en los centros escolares y el confinamiento vivido. 


\section{DIFERENCIAS EN ANSIEDAD EN FUNCIÓN DEL CURSO EN UNIVERSITARIOS}

En el presente estudio no se encontraron diferencias estadísticamente significativas en la variable ansiedad en función del sexo. Ello es congruente con otro estudio previo (Cardona-Arias et al., 2015) en el que no se encontraron en una muestra de estudiantes universitarios diferencias estadísticamente significativas en prevalencia de ansiedad según el sexo.

Es necesario este tipo de evaluaciones sobre la ansiedad y el estrés académico considerando que como plantean Mathews, Koehn, Abtahi y Kerns (2016) los jóvenes con más ansiedad son menos eficaces en lo que a la expresión, entendimiento y aceptación de las emociones se refiere y muestran menos niveles de autoeficacia percibida. Cabe pensar que ello puede afectar de algún modo al proceso de enseñanza aprendizaje, bienestar y rendimiento académico del alumnado.

Puede señalarse la necesidad de realizar talleres que ayuden a los estudiantes al afrontamiento de dichas situaciones estresantes ante los continuos cambios e incluso incertidumbre inicial en las metodologías de la evaluación para adaptarse a las clases no presenciales, el miedo al contagio y la crisis económica que también puede llevar a situaciones personales y familiares con menos recursos financieros para continuar los estudios.

Entre las limitaciones del presente estudio, se encuentran los inconvenientes propios de la aplicación de medidas autoinformadas y la subjetividad que puede existir asociada a las mismas. Además, sería necesario utilizar un tipo de diseño longitudinal que permita un mayor seguimiento de las puntuaciones en la variable ansiedad a lo largo de las distintas etapas evolutivas y niveles educativos.

Futuros estudios también podrían analizar la relación que puede tener la variable que ha sido evaluada en este estudio con otras variables como la inteligencia emocional, la auteoficacia percibida y el empleo de un tipo u otro de estrategias de afrontamiento ante las situaciones que generan estrés y malestar en el alumnado al menos en lo que se refiere al contexto y ambiente universitario. También sería necesario seguir profundizando en mayor medida en el papel que juegan determinadas variables sociodemográficas como el estatus socioeconómico y la influencia de la existencia de problemas en otros ámbitos como puede ser el familiar.

\section{CONCLUSIONES}

Se encontraron diferencias estadísticamente significativas en ansiedad estado y ansiedad rasgo en función el curso. Los datos obtenidos son relevantes para el diseño de acciones e intervenciones psicoeducativas para la prevención del estrés académico y ansiedad, especialmente en esta situación de pandemia, que contribuyan a la mejora del bienestar, calidad de vida y del rendimiento académico en estudiantes universitarios.

\section{REFERENCIAS BIBLIOGRÁFICAS}

Arrieta Vergara K.M., Díaz Cárdenas, S., y González Martínez, F. (2014). Síntomas de depresión y ansiedad en jóvenes universitarios: prevalencia y factores relacionados. Rev Clínica Med Fam, 7(1), 14-22. http://scielo.isciii.es/scielo.php?script=sci_arttext\&pid=s1699695X2014000100003\&lng=es\&nrm=iso\&tlng=es

Caballero-Domínguez, C. y Suarez-Colorado, Y. (2019). Tipologías distintivas de respuestas ante el estrés académico en universitarios: un análisis de clasificación. Duazary, 16(2), 245-57. https://doi.org/10.21676/2389783X.2748

Cardona-Arias J.A., Pérez-Restrepo, D., Rivera-Ocampo, S., Gómez-Martínez, J., y Reyes Á. (2015). Prevalence of anxiety in universitary students. Divers Perspect en Psicol, 11(1), 79-89. http://www.scielo.org.co/scielo.php?script=sci_arttext\&pid=\$1794-99982015000100006

Castillo, C., Chacón, T. y Díaz-Véliz, G. (2016). Ansiedad y fuentes de estrés académico en estudiantes de carreras de la salud. Investigación en Educación Médica, 5, 230-237. Doi: 10.1016/j.riem.2016.03.001

Domínguez-Lara, S. A. (2016). Afrontamiento ante la ansiedad pre-examen y autoeficacia académica en estudiantes de ciencias de la salud. Educación Médica, 99, 1-4. Doi: 10.1016/j.edumed.2016.07.007..

Finney, S. J. y Schraw, G. (2003). Self-efficay beliefs in college statistics course. Contemporary Educational Psychology, 28, 161-186. 
Fonseca-Pedrero, E., Paino, M., Sierra-Baigrie, S., Lemos-Giráldez, S.y Muñiz, J. (2012). Propiedades Psicométricas del "Cuestionario de Ansiedad Estado-Rasgo" (STAl) en Universitarios [Psychometric Properties of the "State-Trait Anxiety Questionnaire"]. Behav. Psychol. Psicol. Conduct., 20, 547-561.

Mathews, B. L., Koehn, A. J., Abtahi, M. M. \& Kerns, K. A. (2016). Emotional competence and anxiety in childhood and adolescence: A meta-analytic review. Clinical Child and Family Psychology Review, 19, 162-184. Doi: 10.1007/s10567-016-0204-3.

Riveros, M., Hernández, H. y Rivera, J. (2007). Niveles de depresión y ansiedad en estudiantes universitarios de Lima Metropolitana. Revista de Investigación en Psicología, 10(1), 91-102.

Spielberger, C. D., Gorsuch, R. L., Lushene, R. E., y Cubero, N. S. (2011). STAl: Cuestionario de ansiedad estado rasgo. TEA Ediciones: Madrid, España.

Spielberger, C. D. (1972). Anxiety as an emotional state. In Spielberger, C. D., (Ed.), Anxiety Behavior (pp. 2349). Academic Press. 
